\title{
Due date optimization in multi-objective scheduling of flexible job shop production
}

\author{
Ojstersek, R. ${ }^{a,}{ }^{*}$, Tang, $M{ }^{b}{ }^{b}$, Buchmeister, B. ${ }^{a}$ \\ ${ }^{a}$ University of Maribor, Faculty of Mechanical Engineering, Maribor, Slovenia \\ ${ }^{b}$ Beijing Jiaotong University, International Center for Informatics Research, Beijing, P.R. China
}

\section{A B S T R A C T}

The manuscript presents the importance of integrating mathematical methods for the determination of due date optimization parameter for maturity optimization in evolutionary computation (EC) methods in multi-objective flexible job shop scheduling problem (FJSSP). The use of mathematical modelling methods of due date optimization with slack (SLK) for low and total work content (TWK) for medium and high dimensional problems was presented with the integration into the multi-objective heuristic Kalman algorithm (MOHKA). The multi-objective optimization results of makespan, machine utilization and due date scheduling with the MOHKA algorithm were compared with two comparative multi-objective algorithms. The high capability and dominance of the EC method results in scheduling jobs for FJSSP production was demonstrated by comparing the optimization results with the results of scheduling according to conventional priority rules. The obtained results of randomly generated datasets proved the high level of job scheduling importance with respect to the interdependence of the optimization parameters. The ability to apply the presented method to the real-world environment was demonstrated by using a real-world manufacturing system dataset applied in Simio simulation and scheduling software. The optimization results prove the importance of the due date optimization parameter in highly dynamic FJSSP when it comes to achieving low numbers of tardy jobs, short job tardiness and potentially lower tardy jobs costs in relation to short makespan of orders with highly utilized production capacities. The main findings prove that multiobjective optimization of FJSSP planning and scheduling, taking into account the optimization parameter due date, is the key to achieving a financially and timely sustainable production system that is competitive in the global market.
\end{abstract}

\author{
ARTICLE INFO \\ Keywords: \\ Flexible job shop scheduling prob- \\ lem (FJSSP); \\ Due date; \\ Makespan; \\ Capacities utilization; \\ Multi-objective optimization; \\ Evolutionary computation; \\ Multi-objective heuristic Kalman \\ algorithm; \\ Simio simulation and scheduling \\ software
}

*Corresponding author: robert.ojstersek@um.si (Ojstersek, R.)

Article history:

Received 15 May 2020

Revised 23 October 2020

Accepted 25 November 2020

\section{References}

[1] Prester, J., Buchmeister, B., Palčič, I. (2018). Effects of advanced manufacturing technologies on manufacturing company performance, Strojniški Vestnik - Journal of Mechanical Engineering, Vol. 64, No. 12, 763-771, doi: 10.5545/sv-jme.2018.5476.

[2] Baker, K.R. (1984). Sequencing rules and due-date assignments in a job shop, Management Science, Vol. 30, No. 9, 1093-1104, doi: $10.1287 / \mathrm{mnsc} .30 .9 .1093$.

[3] Udo, G.J. (1994). A simulation study of due-date assignment rules in a dynamic job shop, Journal of the Operational Research Society, Vol. 45, No. 12, 1425-1435, doi: 10.1057/jors.1994.219.

[4] Gordon, V.S., Proth, J.-M., Chu, C. (2002). Due date assignment and scheduling: SLK, TWK and other due date assignment models, Production Planning \& Control, Vol. 13, No. 2, 117-132, doi: 10.1080/09537280110069621.

[5] Modrák, V., Pandian, R.S. (2010). Flow shop scheduling algorithm to minimize completion time for n-jobs $\mathrm{m}$ machines problem, Tehnički Vjesnik - Technical Gazette, Vol. 17, No. 3, 273-278.

[6] Demir, H.I., Uygun, O., Cil, I., Ipek, M., Sari, M. (2015). Process planning and scheduling with SLK due-date 
assignment where earliness, tardiness and due-dates are punished, Journal of Industrial and Intelligent Information, Vol. 3, No. 3, 173-180, doi: 10.12720/iiii.3.3.173-180.

[7] Ojstersek, R., Brezocnik, M., Buchmeister, B. (2020). Multi-objective optimization of production scheduling with evolutionary computation: A review, International Journal of Industrial Engineering Computations, Vol. 11, No. 3, 359-376, doi: 10.5267/j.ijiec.2020.1.003.

[8] Janes, G., Perinic, M., Jurkovic, Z. (2017). An efficient genetic algorithm for job shop scheduling problems, Tehnički Vjesnik - Technical Gazette, Vol. 24, No. 4, 1243-1247, doi: 10.17559/TV-20150527133957.

[9] Scrich, C.R., Armentano, V.A., Laguna, M. (2004). Tardiness minimization in a flexible job shop: A tabu search approach, Journal of Intelligent Manufacturing, Vol. 15, No. 1, 103-115, doi: 10.1023/B:JIMS.0000010078. 30713.e9.

[10] Simchi-Levi, D., Wu, S.D., Shen, Z.-J.M. (2004). Handbook of quantitative supply chain analysis: Modeling in the ebusiness era, Springer Science \& Business Media, New York, USA.

[11] Wu, Z., Weng, M.X. (2005). Multiagent scheduling method with earliness and tardiness objectives in flexible job shops, IEEE Transactions on Systems, Man, and Cybernetics, Part B (Cybernetics), Vol. 35, No. 2, 293-301, doi: 10.1109/TSMCB.2004.842412.

[12] Chen, B., Matis, T.I. (2013). A flexible dispatching rule for minimizing tardiness in job shop scheduling, International Journal of Production Economics, Vol. 141, No. 1, 360-365, doi: 10.1016/i.ijpe.2012.08.019.

[13] Ojstersek, R., Buchmeister, B. (2020). The impact of manufacturing flexibility and multi-criteria optimization on the sustainability of manufacturing systems, Symmetry, Vol. 12, No. 1, Paper No. 157, doi: 10.3390/sym1201 $\underline{0157 .}$.

[14] Nie, L., Gao, L., Li, P., Li, X. (2013). A GEP-based reactive scheduling policies constructing approach for dynamic flexible job shop scheduling problem with job release dates, Journal of Intelligent Manufacturing, Vol. 24, No. 4, 763-774, doi: $10.1007 / \mathrm{s} 10845-012-0626-9$.

[15] Rajabinasab, A., Mansour, S. (2011). Dynamic flexible job shop scheduling with alternative process plans: An agent-based approach, The International Journal of Advanced Manufacturing Technology, Vol. 54, No. 9-12, 10911107, doi: $10.1007 / \mathrm{s} 00170-010-2986-7$.

[16] Gao, K.Z., Suganthan, P.N., Pan, Q.K., Chua, T.J., Cai, T.X., Chong, C.S. (2014). Pareto-based grouping discrete harmony search algorithm for multi-objective flexible job shop scheduling, Information Sciences, Vol. 289, 76-90, doi: 10.1016/i.ins.2014.07.039.

[17] Ma, D.Y., He, C.H., Wang, S.Q., Han, X.M., Shi, X.H. (2018). Solving fuzzy flexible job shop scheduling problem based on fuzzy satisfaction rate and differential evolution, Advances in Production Engineering \& Management, Vol. 13, No. 1, 44-56, doi: 10.14743/apem2018.1.272.

[18] Na, H., Park, J. (2014). Multi-level job scheduling in a flexible job shop environment, International Journal of Production Research, Vol. 52, No. 13, 3877-3887, doi: 10.1080/00207543.2013.848487.

[19] Xu, H., Bao, Z.R., Zhang, T. (2017). Solving dual flexible job-shop scheduling problem using a bat algorithm, Advances in Production Engineering \& Management, Vol. 12, No. 1, 5-16, doi: 10.14743/apem2017.1.235.

[20] Fu, H.C., Liu, P. (2019). A multi-objective optimization model based on non-dominated sorting genetic algorithm, International Journal of Simulation Modelling, Vol. 18, No. 3, 510-520, doi: 10.2507/ijsimm18(3)co12.

[21] Getachew, F., Berhan, E. (2015). Simulation and comparison analysis of due date assignment methods using scheduling rules in a job shop production system, International Journal of Computer Science \& Engineering Survey, Vol. 6, No. 5, 29-40, doi: 10.5121/ijcses.2015.6503.

[22] Ojstersek, R., Lalic, D., Buchmeister, B. (2019). A new method for mathematical and simulation modelling interactivity: A case study in flexible job shop scheduling, Advances in Production Engineering \& Management, Vol. 14, No. 4, 435-448, doi: 10.14743/apem2019.4.339.

[23] Yin, Y., Wang, D., Cheng, T.C.E. (2020). Due date-related scheduling with two agents: Models and algorithms, Springer Nature Singapore, Singapore, doi: 10.1007/978-981-15-2105-8.

[24] Hajduk, M., Sukop, M., Semjon, J., Jánoš, R., Varga, J., Vagaš, M. (2018). Principles of formation of flexible manufacturing systems, Tehnički Vjesnik - Technical Gazette, Vol. 25, No. 3, 649-654, doi: 10.17559/TV20161012132937.

[25] Moslehi, G., Mahnam, M. (2011). A Pareto approach to multi-objective flexible job-shop scheduling problem using particle swarm optimization and local search, International Journal of Production Economics, Vol. 129, No. 1, 14-22, doi: 10.1016/j.ijpe.2010.08.004.

[26] Zhang, Y., Gong, D.-W., Ding, Z. (2012). A bare-bones multi-objective particle swarm optimization algorithm for environmental/economic dispatch, Information Sciences, Vol. 192, 213-227, doi: 10.1016/j.ins.2011.06.004. 


\title{
Optimizacija roka izdelave pri večkriterijskem prilagodljivem terminiranju proizvodnje po naročilu
}

\author{
Ojstersek, R. ${ }^{\mathrm{a},{ }^{*},}$, Tang, M. $^{\mathrm{b}}$, Buchmeister, B. ${ }^{\mathrm{a}}$ \\ aUniversity of Maribor, Faculty of Mechanical Engineering, Maribor, Slovenia

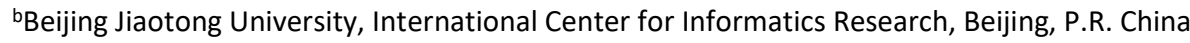

\section{POVZETEK}

Članek predstavlja pomen vključitve matematičnih metod v evolucijsko optimizacijo roka izdelave pri večkriterijskem problemu prilagodljivega terminiranja proizvodnje po naročilu (FJSSP). Za optimizacijo roka izdelave smo za primer nizke in polne zasedenosti strojev predlagali večkriterijski hevristični Kalmanov algoritem (MOHKA). Predlagan pristop je primeren za reševanje srednje težkih do zelo težkih problemov. Sposobnost algoritma MOHKA smo preverili s primerjavo z dvema primerljivima algoritmoma. Primerjali smo tri spremenljivke, čas izdelave, izkoriščenost strojev in razporeditev rokov izdelave. Na podlagi rezultatov smo za terminiranje delovnih nalogov $\mathrm{v}$ okviru FJSSP ugotovili večjo zmogljivost MOHKA v primerjavi z običajnim terminiranjem delovnih nalogov po prednostnih pravilih. Na podlagi rezultatov, pridobljenih z naključno ustvarjenimi podatki, smo potrdili pomembnost terminiranja delovnih nalogov v primeru soodvisnosti parametrov optimizacije. Z uporabo realnih podatkov in simulacijskega programa Simio smo potrdili uporabnost predstavljene metode za realno proizvodnjo. Poudarili smo pomen optimizacije roka izdelave $\mathrm{v}$ dinamičnem FJSSP, kadar so pomembni dejavniki majhno število zamudnih opravil, kratke zamude pri opravilih in nižji stroški zamudnih opravil glede na kratek čas izdelave in visoko izkoriščenost proizvodnih zmogljivosti. Ugotovili smo, da je večkriterijska optimizacija načrtovanja in razporejanja FJSSP, ob upoštevanju roka izdelave, ključ do doseganja finančno vzdržnega in odzivnega proizvodnega sistema, ki je konkurenčen na svetovnem trgu.

\section{PODATKI O ČLANKU}

Ključne besede:

Prilagodljivo terminiranje proiz-

vodnje po naročilu (FJSSP);

Rok izdelave;

Čas izdelave;

Izkoriščenost zmogljivosti;

Večkriterijska optimizacija;

Evolucijsko računanje;

Večkriterijski hevristični Kalmanov algoritem;

Programska oprema za simulacijo in terminiranje Simio

* Kontaktna oseba:

robert.ojstersek@um.si

(Ojstersek, R.)

Zgodovina članka:

Prejet 15. maja 2020

Popravljen 23. oktobra 2020

Sprejet 25. novembra 2020 